\title{
Advantages, Limitations, and Future Trends for Biofabrication Techniques in Tissue Engineering
}

\author{
LvSha Cheng $^{1, a}$, Liu Changyong ${ }^{2}$
}

1. Shen Zhen Polytechnic, School of Mechanical and Electrical Engineering; Shenzhen 518055, China

2. Biomanufacturing Engineering Laboratory, Graduate School at Shenzhen, Tsinghua University

aemail: 49675666@qq.com

Keywords: tissue engineering, biofabrication

\begin{abstract}
Biofabrication techniques facilitate tissue engineering by supplyingtools to construct advanced scaffolds for cell population applied to the assemblingof artificial tissues and organs. Tissue engineering involves the in vitro reconstruction of tissue architectures from nano-scale to macro-scale. In the current review, scaffold fabrication techniques will be classified into nano-scale, micro-scale and macro-scale. Methods for and future trendsof biofabrication techniques will be discussed.
\end{abstract}

\section{Introduction}

Tissue engineering is a promising technology to replace tissue/organ transplantation for the treatment of diseases involving tissue loss and organ failure[1]. Native tissues/organstypically comprise multiple cell types and extracellular matrices (ECM) organized in spatially complex architecture patterns, a set of complex vasculature to provide oxygen and nutrients and/or numerous complex functional units that are essential for normal tissue function. These biological structuresrange from nano-scale to macro-scale. On the nano-scale $(10 \mathrm{~nm} 1000 \mathrm{~nm})$, is the ECM that contains a number of nanostructured components[3]. The diameters of collagen fibers in native ECMrange from $20 \mathrm{~nm}$ to several hundred nanometers. On the micro-scale $(1 \mu \mathrm{m} \sim 100 \mu \mathrm{m})$, the diameter of blood capillaries vastly distributed in various tissues ranges from $6 \mu \mathrm{m}$ to $9 \mu \mathrm{m}$. On the macro-scale $\left(\mathrm{mm}^{\sim} \mathrm{cm}\right)$, the external geometries of tissues, medium-sized and large arteries and veins are in this size range.

At the early stages of the tissue engineering field, researchers assumed that cells were 'smart' and therefore, able to communicate to form native tissues if they were placed in close proximities [4]. This concept was proven to a certain extent, for simple tissue substitutes. However, in most cases, simply placing cells on a scaffold did not result in the formation of complex tissues/organs.These techniques have enabled the incorporation of new features to biomaterial scaffoldsincludingnanoporous features, nanotopographic features, microfluidic features that mimic the microvasculature, and macroporous featuresthat allow cell infiltration.

\section{Scaffold biofabrication techniques}

Understanding the mechanisms by whichisolated cells assemble to become functioning tissues through the use of a scaffold as an instructive microenvironment presenting specific biochemical, structural and mechanical cues to cells to guide them spatially and temporally toward tissue formation[5-7]. Artificial ECM constructs are currently generated via two methods: (1) form an intricate interweaving of protein fibers which are present in native ECM; (2) incorporation of nanoscale topographic features including nanogrooves, nanoposts and nanopits.These structural components provide anoptimal environment for cell adhesion, migration, proliferation and differentiation.To mimic nanofibrous components in native ECM, three approaches to fabricate nanofibrous scaffolds have been developed: electrospinning, thermally-induced phase separation (TIPS) and self-assembly of molecules.

\subsubsection{Electrospinning}


The electrospinning technique was introduced in 1934. This technologyinvolves forcing polymer melt or solution through a nozzle within an external strong electric field to produce nanofibres. The simplicity, scalability, cost-effectiveness and versatility of electrospining have made it the most widely used technique in fabricating nanofibrous scaffolds. Recent innovations in this technique have enabled the technique to produce nanofibrous scaffolds with oriented fiber alignment, multilayer of different polymers, and core-shelled nanostructures. The major limitations of this technology is that it lacksmacroporous features to allow cells to infiltrate into the core of the matrix,nanofibers generated present relatively larger diameters $(>50 \mathrm{~nm})$ than the native ECM (as small as $10 \mathrm{~nm}$ ), the production of fibres require the use of cytotoxic chemical solvents, and 3D structures cannot be precisely fabricated.

\subsubsection{Self-assembly of molecules}

Self-assembly of molecules is a process during which individual molecular components spontaneously assemble into a stable and ordered structure with pre-programmed non-covalent bonds. For example,peptides can bemodified to include functional motif sequences (the cell adhesion motif arginine-glycine-aspartic acid (RGD), a ligand for integrin cell adhesion receptors) to promote cell adherence, differentiation and maturation. The diameter of self-assemblednanofibers can be as short as $10 \mathrm{~nm}$. The limitations of this approach is that it involves complex chemistry, produces scaffolds with a small pore size that limits cell infiltration $(5 \mathrm{~nm}-200 \mathrm{~nm})$, and it cannotproduce oriented fibers.
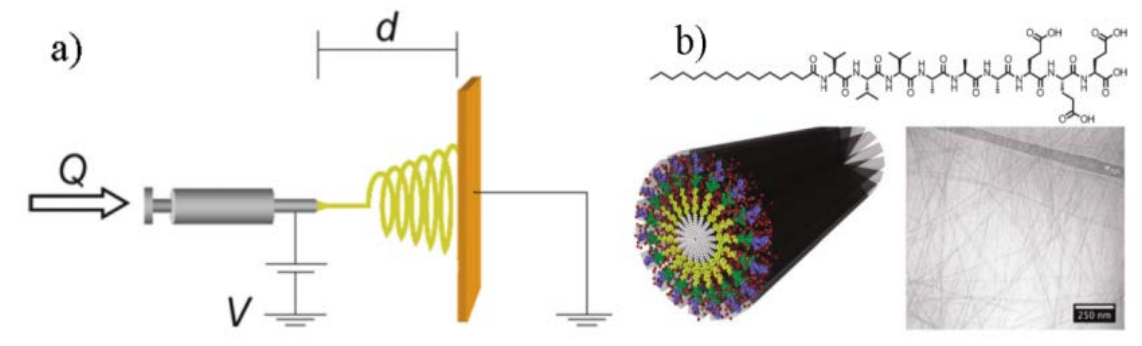

Fig.1 a) Schematic of electrospinning $Q$, flow rate; $d$, distance between plate and needle; $V$, applied voltage. b) Chemical structure of a peptide amphiphile, schematic representation of PA self-assembly into cylindrical nanofibers, and cryogenic transmission electron microscopy (cryoTEM) of assembled aggregates. Adapted with permission from

\subsubsection{Thermally-induced phase separation (TIPS)}

Thermally-induced phase separation (TIPS) has been applied to fabricate tissue engineering scaffolds since the 1990s. Typically, polymers (e.g. poly (L-lactic acid) (PLLA)) are dissolved in an organic solvent, the polymer solution becomes thermodynamically unstable at low temperatures and will separate into two phases: the polymer-rich phase and polymer-lean phase. After freeze drying, the polymer-lean phase is extracted, leaving the polymer-rich phase forming the nanofibrous matrix with diameters of 50nm 500nm. TIPS is a simple approach to fabricate scaffolds with high porosity (>95\%). However, the approach is only available for crystalline polymers and cannot currently be used to fabricate scaffolds with oriented alignment.

\subsubsection{Nanotopography fabrication techniques}

Nanoscale geometric features, i.e.nanogrooves, nanoposts, nanopit arrays, nanofibers, nanospheres, and vertical nanotubes, can direct cell adhesion, proliferation, migration, differentiation, and morphology. Nanotopographyfabrication techniquescan be grouped into two categories: 1.) techniques to produce ordered nanotopographies, and 2.) techniques to produce unordered nanotopographies. The former category includes replica molding of biomaterials (PDMS, synthetic biopolymers and natural biopolymers) using photolithographic techniques and electron beam lithography. Nanotopography fabrication techniques are currently primarily used to generate surface nanotopographic features in two-dimensional systems; the expansion to three-dimensional systems remains a challenge.

\subsection{Scaffold biofabrication techniques at micro-scale}

Any tissue that is several hundred microns in depth requires a vascular network to provide 
oxygen and nutrients to cells [2, 4]. The difficulty of vascularization is a prevailing issue in the engineering of large tissues. Originally, vascularization relied on the infiltration of host microvessels into implanted constructs. However, limited infiltration depth and relatively slow neovascularisationmay cause many of the cells within the construct to die due to lack of an immediate blood supply. Therefore, prefabricated vascular systems embedded in implanted constructs are necessary [4]. To achieve vascularised engineered tissue biofabrication techniques have incorporated micro-scale artificial blood vessels into constructs..

\subsection{Scaffold biofabrication techniques at macro-scale}

Conventional scaffold fabrication techniques e.g., solvent casting and particle leaching, gas foaming, and fiber bondingare unable to control individual pore size, shape and distribution, interconnectivity and porosity of scaffolds. To overcome these limits, bioprinting techniques are developed and dramatically enhanced the design flexibility and enabled the fabrication of scaffold with arbitrarily complex internal architecture and external shape at a spatial resolution of approximately $100 \mu \mathrm{m}$. These techniques include stereolithography (SLA), fused deposition modeling (FDM) and selective laser sintering (SLS), and three-dimensional printing (3DP).

\section{Future trends}

\subsection{Fabricate multi-scale featured structures}

The hierarchical structures of native tissues indicate that biofabrication techniques that only capable of fabricating structures at single size scale will achieve limited success as clinically-viable artificial organ constructs. Multi-scale featured scaffolds combining nanoporous features, microarchitectures and macroporous features is the approach that will allow construction of physiological artificial tissues.3D scaffolds that were several millimeters in diameter were produced using 2PP indicating that this technique is potentially applicable to large scale production of tissue engineered scaffolds. Another approach is to combine biofabrication techniques at different size scales in one procedure to fabricate multi-scale featured structures. A typical technique developed in our lab is the low temperature deposition process. By combining rapid prototyping, porogen leaching, and thermal-induced phase separation, scaffolds with hierarchical structures of three-level pore sizes can be fabricated (pore size from $1 \mu \mathrm{m}$ to $10 \mu \mathrm{m} ; 10 \mu \mathrm{m}$ to $100 \mu \mathrm{m}$ and over $100 \mu \mathrm{m}$ ).

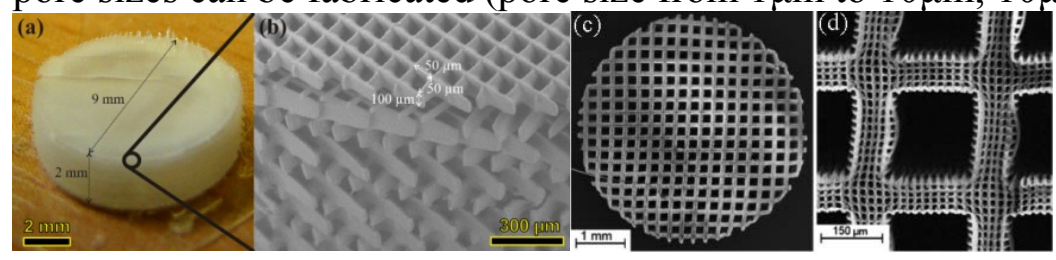

Fig.3 examples of large scaffold with dimensions up to several millimeters; a) and b) SZ2080 polymer, cylindrical scaffold with the diameter of $9 \mathrm{~mm}$ and height of $2 \mathrm{~mm}$, adapted from; c) and d) ORMOCER $^{\circledR}$, cylindrical microporous and macroporous combined scaffold with diameter of $5 \mathrm{~mm}$ and height of $1.2 \mathrm{~mm}$

\subsection{Scaffold-free cellular construct fabrication techniques}

One of the primary limitations of the cells-on-a-scaffold approach is that arbitrary cell distribution cannot facilitate cell-cell contact and the formation of organized arrangement of multiple cell types and tissue architectures.As a result, some researchers developed "scaffold-free" tissue engineering approaches directly using cells or using cell-laden hydrogels as building blocks to fabricate 2D and 3D cellular constructs. These "scaffold-free" approaches can be classified into several categories in terms of the building units: (1) single cellsusing cell printing technologies, but the printing efficiency and the post printing cell viability need to be improved before it becomes a reliable tool to apply in tissue engineering; (2) microdroplets of cells, the feature is the uncertain number of cells contained in the printed microdroplets, (cells may be damaged due to heat exposure, shear stress, and exposure to laser); (3) multicellular microtissue (usually around $300 \mu \mathrm{m}-500 \mu \mathrm{m}$ in diameter), the key steps include the production of microtissues(the assembly of microtissues, and the precision still needs to be improved); (4) cell-laden hydrogel filaments(the filament width is up 
to over $200 \mu \mathrm{m}$ and the precision still needs to be improved).; (5) 2D cell sheets,.
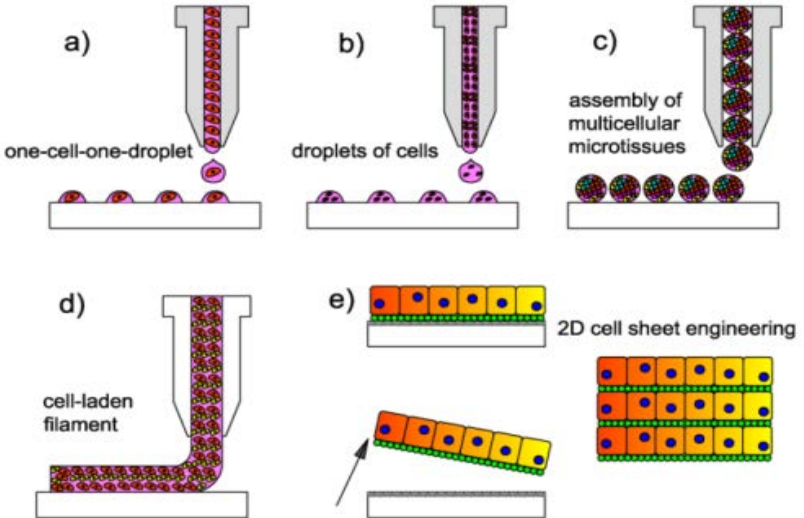

Fig. 4 Cellular construct fabrication techniques using a) single cell as building blocks; b) microdroplets of cells as building blocks; c) multicellular microtissue as building blocks; d) cell-laden filament as building blocks; e) 2D cell sheets as building blocks

\section{References}

[1] LANGER R, VACANTI J P. Tissue Engineering. Science, 1993, 260: 920-926

[2] GRIFFITH L G, NAUGHTON G. Tissue engineering--current challenges and expanding opportunities. Science, 2002, 295: 1009-1014

[3] STEVENS M M, GEORGE J H. Exploring and engineering the cell surface interface. Science, 2005, 310: 1135-1138

[4] KHADEMHOSSEINI A, VACANTI J P, LANGER R. Progress In Tissue Engineering. Sci Am, 2009, 300: 64-+

[5] SZENTIVANYI A, CHAKRADEO T, ZERNETSCH H, GLASMACHER B. Electrospun cellular microenvironments: Understanding controlled release and scaffold structure. Advanced drug delivery reviews, 2011, 63: 209-220 